\title{
Design of RF Diplexer for Mobile Communication
}

\author{
R.Brinda \\ Electronics and Communication \\ Mepco Schlenk Engineering College \\ Sivakasi,India
}

\author{
P.Anisha Parveen \\ Electronics and Communication \\ Mepco Schlenk Engineering College \\ Sivakasi, India
}

\begin{abstract}
In this paper, a matching circuit for RF diplexer for mobile communication is proposed. Most wanted bands in wireless technologies like GSM and DCS is used. Both bands are designed by using stepped impedance resonators with single order and matching between this two are implemented by Yjunction method. Cutoff frequency for GSM and DCS band are $925 \mathrm{MHz}$ and $1795 \mathrm{MHz}$. Microstrip Technology is proposed because it provides a better insertion loss, better return loss and good isolation between the passbands. The isolation between two operating bands is better than $-50 \mathrm{~dB}$. The overall insertion loss obtained in diplexer is $-0.4 \mathrm{~dB}$. The overall return loss is less than $-20 \mathrm{~dB}$.
\end{abstract}

\section{Keywords}

Diplexer, Mobile Communication, Stepped Impedance Resonator (SIR).

\section{INTRODUCTION}

Diplexer plays a important role in wireless communications. Industry demand us to design a single wide band compact antenna which will cover all the CDMA ,WCDMA,UMTS and PCS bands with good gain. One of the major advantages of this diplexer is non- requirement of extra jumper cable and connector. Therefore it is economical and also avoids cable insertion loss. Moreover, Diplexer is designed should ensure desired isolation between the dual pass bands. The matching circuit design of lowpass-bandpass [1], [2] may be challenge in conventional designs. Coupled line bandpass topology is used. It requires more space [1]. Generally the junction circuit design is more complex and critical part of the Diplexer because the matching circuit may provides easy independent design for each filter.[3]-[6]...Folded coupled-line structure proposed in [4] and dual-mode Stripline ring resonators are used to produce transmission zeros to improve the selectivity of the Diplexer. Skew symmetric feed structure is used. But it does not concentrate on the matching circuit. The Branch line resonator can also act as diplexer junction. For dual-band filter structure is for providing the required resonance at the centre frequency of each passband [8].

For better Matching between the filter, Two BPF is combined with $\mathrm{T}$ junction. It can be used for several mobile applications. It consists of compact hybrid resonator. It is larger in size and complex to design. Coupling structures with both Chebyshev and quasi-elliptic freq responses presented to achieve dual\&triple-band without a significant increase in circuit size.[7] some extra coupled resonator section can added for increasing the degrees of freedom in extracting coupling coefficient of a multiband filter. It is Flexible design process. But it is more complex. Many application need High Isolation and selectivity. Filters with asymmetric responses are very suitable for duplexer applications, because it make the use of band-width more efficient [3]. Coplanar-waveguide diplexer is using in planar lumped-elements to realize lowpass and bandpass filter in diplexer structure. Cpw-cps cross junction is proposed. [9]. It requires MMIC components. Two bandpass filters are used with very large dielectric constant. The filter structure is based on the stepped impedance resonator(SIR). So. Spurious response is effectively reduced. The T-shaped resonator has an extremely small frequency ratio using microstrip with practical line width. T-shaped gives better isolation.[3].But Stripline technology is very difficult to fabricate and design. A compact diplexer is implemented by using Slotline hairpin Stepped Impedance Resonator (SIR) in ground plane [10]. Microstrip-Slotline transition is used to split the signal without extra matching circuits and thereby economizes the circuit size. To achieve high isolation and compact size diplexer, the variable frequency response of stepped-impedance resonators (SIR) is applied to realize the diplexer if their fundamental and the first spurious resonant frequency are properly assigned [11]. Microstrip diplexers, due to the low profile, light weight, low cost, and easy integration with other front-end circuitry, have recently become more and more popular [14][15]. It is, however, a challenging task to design microstrip diplexers with low inband losses for both channels and good isolation between them while keeping a small size.

The matching network and combining circuit ensure that both filters match the antenna and have good isolation between them. The T-junction may be the most popularly used combining circuit. The length and width of its two branches must be chosen carefully [16], [5], allowing each filter to match the antenna and introduce an open circuit at the middle band of the other. T-junctions with open stubs [17] or a step impedance transformer [18] have been developed to improve the performance..

\section{PROPOSED METHODOLOGY}

So we proposed a diplexer design with two bandpass filter which is designed using Stepped Impedance Resonator method and matching between these two bandpass filter using Y-junction matching devices thereby we can economizes the circuit size. Diplexer designed for the base station antenna which allows combination and separation of the signals in band 1 and band 2 wireless bands. To minimize band interreaction, the inputs are all isolated and have minimal insertion loss over their respective frequency bands. The design ensures low-passive intermodulation. An efficient Diplexer needs high isolation between two passband frequencies to avoid interference of signal from one port to another. Each filter at two ports should act as a short circuit in its pass band and open circuit at other passband. There should be Minimum insertion loss to minimize power loss at its two ports.

\section{DESIGN OF BANDPASS FILTERS}

GSM-900 (890-960 MHz) band and DCS-1800 (1710-1880 $\mathrm{MHz}$ ) band are the most wanted bands in mobile communication so we can used these two bands to design a diplexer. Chebyshev approximation is used because, In Chebyshev approximation, steep roll-off rate in its stop band frequency and tolerable ripple level in pass band by using very less number of filter reactive elements can be achieved, and also low insertion loss and less filter size, due to usage of 
less number of reactive elements..The order of the filter is more important while considering the size of the circuit.. It is related to the number of components in the filter, because of its cost and its physical size. So here we prefer single order is used.

Bandpass filter is designed by using one Transmission line and two step-impedance open stubs.[19]. In this design, a step-impedance open stub can produce two transmission zeros loaded at each side of the central frequency. The bandwidth of the filter is controlled by the positions of the transmission zeros produced by the step-impedance open stub. By introducing two step-impedance open stubs to the input and output port of the transmission line which resonates at the desired centre frequency, we can achieve filter with good performances of low loss, good selectivity, and sharp attenuation characteristics.

In Figure 6.1 shows the configuration of a step-impedance open stub. The two sections of the resonator are quarterwavelength (i.e. $\left.\lambda_{\mathrm{o}} / 4\right)$ long at the central frequency of the filter and have impedances of $Z_{1}$ and $Z_{2}$ respectively. The impedances of input and output ports in Figure below are assumed to be $50 \Omega$. A pair of transmission zeros can be produced at the frequency $\mathrm{f}_{1}$ and $2 \mathrm{f}_{0}-\mathrm{f}_{1}\left(\mathrm{f}_{0}\right.$ is the central frequency of the filter). ). By investigating the positions (i.e. the frequencies) of the transmission zeros using transmission line theory, the following equation can be obtained as follows[19]:

$$
\theta_{1}=\arctan \sqrt{\frac{Z_{2}}{Z_{1}}}
$$

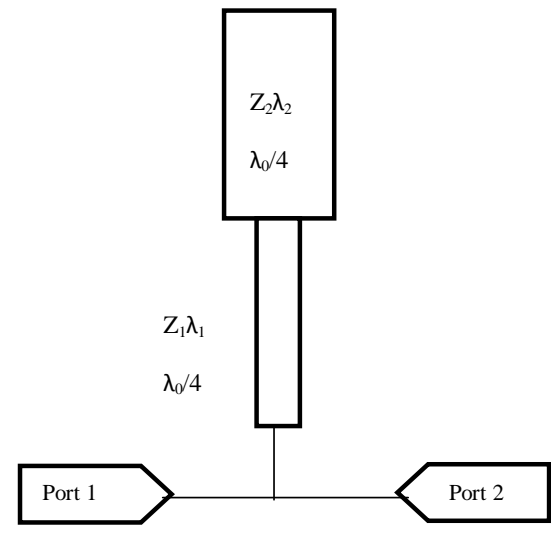

Fig1 Stepped impedance open stub bandpass filter

where $\theta_{1}$ represents the electrical length at which the transmission zero occurs. $f_{1}$ and $\theta_{2}$ should satisfy the following relation:

$$
f_{1}=\frac{2 \theta_{1}}{\pi} f_{0}
$$

The above formula reveals that the positions of transmission zeros produced by the step-impedance open stub are only determined by the impedance ratio, $\mathrm{Z}_{2} / \mathrm{Z}_{1}$, of the two sections of the resonator. The line-width and length of the transmission-line stage are calculated while the electrical length of the stage is $\lambda_{0} / 4$. In the following design, a substrate with a dielectric constant of 10.2 and a thickness of $0.635 \mathrm{~mm}$ is used.

In order to improve the characteristics of the filter's stop band, it is designed by introducing two similar stepimpedance open stubs to both sides of a coupled-line stage. The step-impedance open stubs produce two transmission zeros near the pass band of the filter. As a result, sharp attenuations are obtained near the pass band. The sharp attenuation also gives required isolation between two passbands.[19].A classical formalism can be used to synthesize a nth-order filter. The resonators being defined by a proper coefficient, the designer has just to calculate the characteristic impedances of quarter wavelength admittance inverters defined as follows [22][19]

$$
\frac{Z_{c j, j+1}}{Z_{0}}=\frac{1}{J_{j, j+1}}
$$

where

$$
\begin{aligned}
& J_{01}=\sqrt{\frac{G_{a} \cdot b_{1} \cdot \omega}{\omega_{1} \cdot g_{0} \cdot g_{1}}} \\
& J_{j, j+1}=\frac{\omega}{\omega_{1}} \sqrt{\frac{b_{j} \cdot b_{j+1}}{g_{j} \cdot g_{j+1}}} \\
& J_{n, n+1}=\sqrt{\frac{G_{a} \cdot b_{n} \cdot \omega}{\omega_{1} \cdot g_{n} \cdot g_{n+1}}}
\end{aligned}
$$

In these formulas, the coefficients $g_{j}$ are the Tchebyscheff coefficients of the equivalent low-pass filter prototype. The parameter $\omega_{1}$ is the cut-off frequency of the low-pass prototype and $\mathrm{G}_{\mathrm{a}}$ and $\mathrm{G}_{\mathrm{b}}$ the terminating conductances of the circuit. Finally, is defined as the fractional bandwidth. As we use an admittance inverter, the coefficients can be used as a dimensionless constant. In this way, a tuning parameter is thus introduced. This parameter can be chosen arbitrarily, mostly depending on the achievable characteristic impedance values of the technology in use.

\subsection{Design of BPF for GSM-900}

By using these formulas, we can able to calculate the impedance and electrical length of each stubs present in the BPF. For size reduction, here we design a BPF with first order. Calculate impedance and electrical length value for first BPF(890-960 MHz).After Calculation convert those electrical parameter to physical parameter values by using 'Linecalc' which is provided by Agilent Technologies Inc.

Table 1. Section values for BPF (890- $960 \mathrm{MHz})$

\begin{tabular}{|l|c|c|c|c|}
\hline Segment & $\begin{array}{c}\text { Impeda } \\
\text { nce }\end{array}$ & $\begin{array}{c}\text { Impedance } \\
\text { values }\end{array}$ & Width & Length \\
\hline \multirow{3}{*}{$\begin{array}{l}\text { STEP } \\
\text { IMPED }\end{array}$} & $\mathrm{Z}_{11}$ & $18.031 \Omega$ & $3.033 \mathrm{~mm}$ & $16.521 \mathrm{~mm}$ \\
\cline { 2 - 5 } & $\mathrm{Z}_{12}$ & $60.568 \Omega$ & $0.455 \mathrm{~mm}$ & $9.743 \mathrm{~mm}$ \\
\cline { 2 - 5 } & $\mathrm{Z}_{21}$ & $18.031 \Omega$ & $3.033 \mathrm{~mm}$ & $16.521 \mathrm{~mm}$ \\
\hline
\end{tabular}




\begin{tabular}{|l|c|c|c|c|}
\hline ANCE & $Z_{22}$ & $60.568 \Omega$ & $0.455 \mathrm{~mm}$ & $9.743 \mathrm{~mm}$ \\
\cline { 2 - 5 } STUBS & $Z_{\mathrm{c} 01}$ & $24.668 \Omega$ & $1.965 \mathrm{~mm}$ & $31.452 \mathrm{~mm}$ \\
\cline { 2 - 5 } & $Z_{c 02}$ & $24.668 \Omega$ & $1.965 \mathrm{~mm}$ & $31.452 \mathrm{~mm}$ \\
\hline
\end{tabular}

\subsection{Design of BPF for DCS-1800}

The same procedure which is used in the first BPF is followed for second BPF with frequency band (1710-1880 MHz). Also, tabulate the physical parameter for second BPF is shown in Table 2.

Table 2. Section values for BPF (1710-1880 MHz)

\begin{tabular}{|c|c|c|c|c|}
\hline $\begin{array}{c}\text { Segment } \\
\text { s }\end{array}$ & $\begin{array}{c}\text { Impeda } \\
\text { nce }\end{array}$ & $\begin{array}{c}\text { Impedance } \\
\text { values }\end{array}$ & Width & Length \\
\hline \multirow{4}{*}{\begin{tabular}{c} 
STEP \\
IMPED \\
ANCE \\
\cline { 2 - 5 }
\end{tabular}} & $\mathrm{Z}_{11}$ & $15.696 \Omega$ & $3.601 \mathrm{~mm}$ & $8.512 \mathrm{~mm}$ \\
\cline { 2 - 5 } STUBS & $\mathrm{Z}_{12}$ & $30.025 \Omega$ & $1.465 \mathrm{~mm}$ & $6.080 \mathrm{~mm}$ \\
\cline { 2 - 5 } & $\mathrm{Z}_{22}$ & $15.696 \Omega$ & $3.601 \mathrm{~mm}$ & $8.512 \mathrm{~mm}$ \\
\cline { 2 - 5 } & $Z_{c 01}$ & $15.594 \Omega$ & $3.631 \mathrm{~mm}$ & $14.997 \mathrm{~mm}$ \\
\cline { 2 - 5 } & $Z_{c 02}$ & $15.594 \Omega$ & $3.631 \mathrm{~mm}$ & $14.997 \mathrm{~mm}$ \\
\hline
\end{tabular}

Both BPF are designed using Microstrip structure using the substrate RT/Duroid $6010 \mathrm{LM}$ which is having dielectric constant as 10.2 and substrate thickness $t$ is $0.635 \mathrm{~mm}$. copper is conductor with thickness $17 \mu \mathrm{m}$. Consider the tangent value as $(\operatorname{Tan} \delta$ ) is 0.002 .

Here we use RT/Duroid 6010LMsubstrate. RT/Duroid 6006 is also available. This substrate provided by ROGERS Corporation.RT/Duroid 6006/6010LM microwave laminates feature ease of fabrication and stability in use. They have tight dielectric constant and thickness control, low moisture absorption, and good thermal mechanical stability.

\section{DESIGN OF MATCHING CIRCUIT}

The Matching network and combining circuit ensure that both filters match the antenna and have good isolation between them. The T-junction may be the most popularly used combining circuit. The length and width of its two branches must be chosen carefully.

The T-shaped resonator is composed of a three-section transmission line. Each section has an adjustable characteristic impedance and length. The most intuitive approach is to combine two BPFs with a T-junction /Y-junction. The method, which features the Filter A (Filter B) an open-circuitload shunted to the Filter B (Filter A) at the frequency of the latter, is adopted to Figure the dimensions of the connecting line for reducing the mutual loading effect between the Filters. We calculate these three impedances by using T- Matching networks impedance formulas. Consider this as a LCC network. [23][24]

Select the desired bandwidth and calculate $\mathrm{Q}=\mathrm{f} / \mathrm{BW}$
Where $\mathrm{f}$ is operating frequency and $\mathrm{BW}-$ Bandwidth

- $\quad$ Calculate $\mathrm{X}_{\mathrm{L}}=\mathrm{Q} \cdot \mathrm{R}_{\mathrm{g}}$

- Calculate $X_{c 2}=R_{L} \sqrt{\frac{R_{g}\left(Q^{2}+1\right)}{R_{L}-1}}$

- Calculate

$$
X_{c 1}=R_{L} \sqrt{\frac{R_{g}\left(Q^{2}+1\right)}{Q\left(Q R_{L} /\left(Q R_{L}-X_{C 2}\right)\right.}}
$$

- Calculate the inductance and capacitances

$$
L=\frac{X_{L}}{2 \pi f} \quad C=\frac{1}{2 \pi f X_{C}}
$$

Width and length of each impedance is calculated by 'Linecalc'

Table 3. Section values for $\mathrm{Y}$-junction

\begin{tabular}{|c|c|c|c|c|}
\hline Segment & $\begin{array}{c}\text { Imped } \\
\text { ance }\end{array}$ & $\begin{array}{c}\text { Impedance } \\
\text { values }\end{array}$ & Width & Length \\
\hline & $\mathrm{Z}_{\mathrm{a}}$ & $64.330 \Omega$ & $0.3124 \mathrm{~mm}$ & $8.990 \mathrm{~mm}$ \\
$\begin{array}{c}\text { Y- } \\
\text { Junction } \\
\text { STUBS }\end{array}$ & $\mathrm{Z}_{\mathrm{b}}$ & $45.891 \Omega$ & $0.6875 \mathrm{~mm}$ & $24.252 \mathrm{~mm}$ \\
\cline { 2 - 5 } & $\mathrm{Z}_{\mathrm{c}}$ & $2.657 \Omega$ & $3.825 \mathrm{~mm}$ & $\mathbf{4 . 8 6 0} \mathrm{mm}$ \\
\hline
\end{tabular}

\section{DESIGN OF DIPLEXER}

The first step is designing the first Bandpass filter to get the desired output. The Bandpass Filter is designed for the frequency band of 890-960 MHz. The design should ensure that the stop band lies on the low frequency band of the diplexer. For a single bandpass filter for GSM Band (890-960 $\mathrm{MHz}$ ) the desired bandwidth (890 MHz-960 MHz) has given return loss (S11) of $-45.683 \mathrm{~dB}$ at $925 \mathrm{MHz}$. The corresponding insertion loss (S21) obtained is $-0.08 \mathrm{~dB}$ at $890-960 \mathrm{MHz}$. The isolation obtained at the stop band is -40 $\mathrm{dB}$ at $0.7 \mathrm{GHz}$ and $-55 \mathrm{~dB}$ at $1.35 \mathrm{GHz}$.

The next step is designing the second Bandpass filter to get the desired output. The Bandpass Filter is designed for the frequency band of 1710-1880 MHz. The desired bandwidth (1710 MHz-1880 MHz) has given return loss (S11) of $25.648 \mathrm{~dB}$ at $1.795 \mathrm{GHz}$.The resultant insertion loss obtained is nearly $-0.1 \mathrm{~dB}$ at $1710-1880 \mathrm{MHz}$. The isolation obtained at the stop band is $-37 \mathrm{~dB}$ at $1.5 \mathrm{GHz}$ and $-45 \mathrm{~dB}$ at $2.1 \mathrm{GHz}$ 


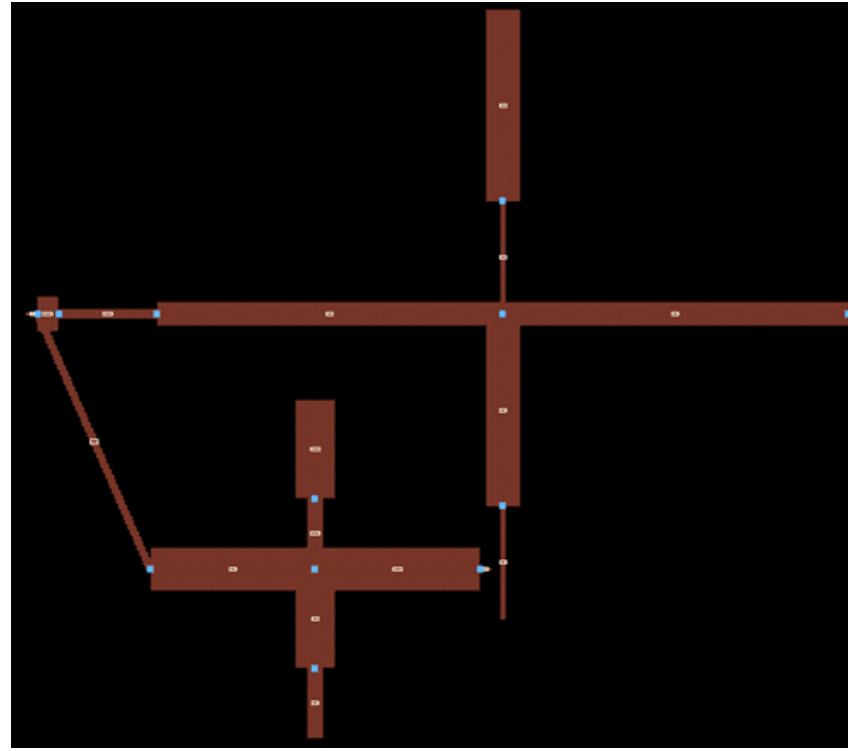

Fig 2. Design of Diplexer in Layout Window

For designing the Diplexer, we need to match these two BPF. T-Junction/Y-junction resonator is used to design the Diplexer. The section from the Table 3 is using to design the Y- junction resonator is shown in the Fig 2. The Diplexer is simulated for a frequency range of $0.5 \mathrm{GHz}$ to $2.2 \mathrm{GHz}$. All the scattering parameters are simulated and their response is shown in Fig 3.

The Matching between the two BPF is the final step for this diplexer. There are several methods to integrate a diplexer. The most important section is the T-junction. The T-junction serves to divide the signal of the entire bandwidth to appropriate bands .The length and width of the Diplexer is given as Length $=7.32 \mathrm{~cm}$; Width $=6.32 \mathrm{~mm}$.

The entire diplexer is simulated and the result is shown in Figure 3. The observations of the simulated results are as follows: .The overall return loss at the low frequency band $(890-960 \mathrm{MHz})$ is obtained as $-23.6 \mathrm{~dB}$. The insertion loss has been reduced to $-0.2 \mathrm{~dB}$ for the same band. The overall return loss at the High frequency band $(1710-1880 \mathrm{MHz})$ is obtained as $-21.49 \mathrm{~dB}$. The insertion loss obtained for this band is -0.3 $\mathrm{dB}$. The higher band is perfectly isolated from the lower band. The isolation obtained is more than $-50 \mathrm{~dB}$. The specification has been achieved from the simulated results of the diplexer.

\section{CONCLUSION}

In this paper, the stepped impedance BPF for GSM band and DCS band were designed using Microstrip separately and analyzed using ADS 2009.The Bandwidth is obtained for both GSM900(890-960 MHz) and DCS1800(1710-1880 MHz) effectively. In order to get required inter band isolation, the BPF are designed and open circuited step impedance stubs were placed at the input and output. Because of the structure wide stopband and excellent interband isolation of above -50 was achieved. The insertion loss obtained is less than $-0.4 \mathrm{~dB}$. The overall return loss is less than $-18.5 \mathrm{~dB}$. The two filters are integrated to form a Diplexer which can be used in high power applications. This integrated system fulfilling all the specifications. It can also be used in dual band cellular networks, common antenna sharing and in-building coverage systems to share two channels using a common antenna.

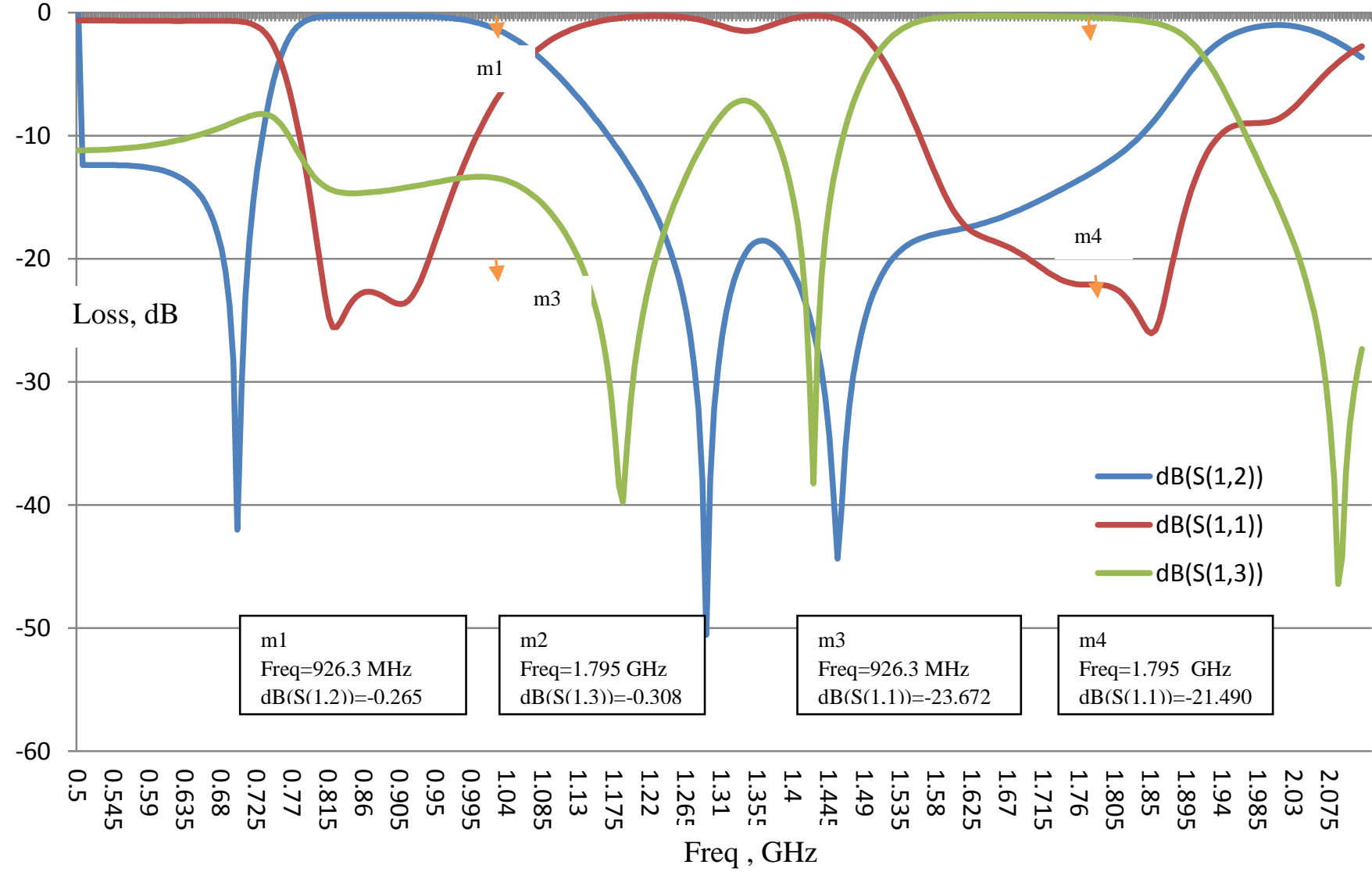

Fig. 3 Simulated result for diplexer structure. 


\section{REFERENCES}

[1] Pu-Hua Deng and Jen-Tse Tsai, "Design of Microstrip Lowpass-Bandpass Diplexer", IEEE Microw. Wireless Compon. Lett., vol. 23, no. 7, pp. 332-334, Jul. 2013.

[2] M. H. Capstisk, "Microstrip lowpass-bandpass diplexer topology," Electron. Lett., vol. 35, no. 22, pp. 19581960, Oct. 1999

[3] A. F. Sheta, J. P. Coupez, G. Tanne, S. Toutain, and J. P. Blot, "Miniature microstrip stepped impedance resonator bandpass filters and diplexers for mobile communications," in IEEE MTT-S Int. Dig., Jun. 1996, pp. 607-610.

[4] C.M. Tsai, S. Y. Lee, C. C. Chuang, and C. C. Tsai, "A folded coupled line structure and its application to filter and diplexer design," in IEEE MTT-S Int. Dig., Jun. 2002, pp. 1927-1930.

[5] T. Yang, P. L. Chi, and T. Itoh, "High isolation and compact diplexer using the hybrid resonators," IEEE Microw. Wireless Compon. Lett.,vol. 20, no. 10, pp. 551-553, Oct. 2010

[6] P. H. Deng and J. H. Jheng, "A switched reconfigurable high-isolation dual-band bandpass filter," IEEE Microw. Wireless Compon. Lett., vol. 21, no. 2, pp. 71-73, Feb. 2011.

[7] C. F. Chen, T. Y. Huang, and R. B. Wu, "Design of dualand triple-passband filters using alternately cascaded multiband resonators," IEEE Trans. Microw. Theory Tech., vol. 54, no. 9, pp. 3550-3558, Sep. 2006.

[8] P. H. Deng and H. H. Tung, "Design of microstrip dualpassband filter based on branch-line resonators," IEEE Microw.Wireless Compon. Lett., vol. 21, no. 4, pp. 200 202, Apr. 2011.

[9] Y. S. Lin and C. H. Chen, "Lumped-element coplanarwaveguide diplexer," in Proc. Eur. Microw. Conf., Sep. 2002, pp. 1-4.

[10] Haiwen Liu, Wenyuan Xu, Zhichong Zhang, and Xuehui Guan, "Compact Diplexer Using Slotline Stepped Impedance Resonator ",IEEE Microw. Wireless Compon. Lett., vol. 23, no. 2, pp. 75-77, Feb. 2013.

[11] C. F. Chen, T. Y. Huang, C. P. Chou, and R. B. Wu, "Microstrip diplexers design with common resonator sections for compact size, but high isolation," IEEE Trans., Microw. Theory Tech., vol. 54, no. 5, pp. 19451952, May 2006.

[12] Y. Toutain, C. Person, and J. P. Coupez, "Design and implementation of a compact microstrip Tx/Rx diplexer for UMTS equipments," in Proc. Int. MIKON'02 Conf., 2002, pp. $187-190$

[13] C.-F. Chen, T.-Y. Huang, T.-M. Shen, and R.-B. Wu, "A miniaturized microstrip common resonator triplexer without extra matching network," in Proc. Asia-Pacific Microw. Conf., 2006, pp. 1439-1442.

[14] S. Srisathit, S. Patisang, R. Phromloungsri, S. Bunnjaweht, S. Kosulvit, and M. Chongcheawchamnan, "High isolation and compact size microstrip hairpin diplexer," IEEE Microw. Wireless Compon. Lett., vol. IS, no. 2, pp. 101-103, Feb. 2005.

[15] D. Puttadilok, D. Eungdamrong, and S. Amomsaensak, "A Microstrip diplexer filter using stepped-impedance resonators," SICE Annual Conference, Aug. 2008, pp $59-62$

[16] J. Konpang, "A compact diplexer using square open loop with stepped impedance resonators," in IEEE Radio Wireless Symp. Dig., Jan. 2009, pp. 91-94.

[17] C. Collado, J. Pozo, J. Mateu, and J. M. O’Callaghan, "Compact duplexer with miniaturized dual loop resonator," in Proc. Eur. Microw. Conf., Oct. 2005, vol. 1, [CD ROM].

[18] E. E. Djoumessi, "Compact packaged diplexer based on highly selective dual-mode bandpass filter," IEEE Microw. Mag., vol. 12, no. 1, pp. 89-93, Feb. 2011.

[19] Cendric Quendo.E.Rius and Christian person, "Narrow Bandpass Filter Using Dual-Behavior Resonators",IEEE Trans., Microw. Theory Tech., vol. 51, no. 3, pp. 734743, Mar. 2003

[20] P. H. Deng, C. H. Wang, and C. H. Chen, "Compact microstrip diplexers based on a dual-passband filter," in Proc. Asia-Pacific Microw. Conf. Dig., Dec. 2008, pp. $1228-1232$

[21] T. Yang, P. L. Chi, and T. Itoh, "Compact quarter-wave resonator and its applications to miniaturized diplexer and triplexer," IEEE Trans., Microw. Theory Tech., vol. 59, no. 2, pp. 260-269, Feb. 2011.

[22] David Pozar-Microwave Engineering. Newyork: wiley.1998

[23] Ming-Lin Chuang and Ming-Tien Wu, "Microstrip Diplexer Design using common T-Shaped Resonator," IEEE Microw.Wireless Compon. Lett., vol. 21, no. 11, pp. 583-585, Nov. 2011

[24] G.L. Mattaei, L.Young, and E.M.T. Jones, "Microwave Filters,Impedance-Matching Network, and coupling Structures. Norwood, MA: Artech House, 1980. 